Corrigendum

\title{
Corrigendum to "Solid Pseudopapillary Neoplasm of the Pancreas with High-Grade Malignant Transformation Involving p16-RB Pathway Alterations"
}

\author{
Kodai Tomioka $\left(\mathbb{D},{ }^{1}\right.$ Nobuyuki Ohike $\left(\mathbb{D},{ }^{2}\right.$ Takeshi Aoki, ${ }^{1}$ Yuta Enami, ${ }^{1}$ Akira Fujimori, ${ }^{1}$ \\ Tomotake Koizumi, ${ }^{1}$ Tomokazu Kusano, ${ }^{1}$ Koji Nogaki, ${ }^{1}$ Yoshihiko Tashiro, ${ }^{1}$ Yusuke Wada, ${ }^{1}$ \\ Tomoki Hakozaki, ${ }^{1}$ Hideki Shibata, ${ }^{1}$ Takahito Hirai, ${ }^{1}$ Tatsuya Yamazaki, ${ }^{1}$ \\ Koichiro Fujimasa, ${ }^{1,2}$ Tomoko Norose, ${ }^{2}$ Tomohide Isobe, ${ }^{2}$ and Masahiko Murakami ${ }^{1}$ \\ ${ }^{1}$ Division of Gastroenterological and General Surgery, Department of Surgery, Showa University, Shinagawa, 1-5-8 Hatanodai, \\ Shinagawa, 142-8666 Tokyo, Japan \\ ${ }^{2}$ Department of Pathology and Laboratory Medicine, Showa University Fujigaoka Hospital, 1-30 Fujigaoka, Aoba-Ku, Yokohama, \\ 227-8501 Kanagawa, Japan \\ Correspondence should be addressed to Nobuyuki Ohike; ohike@med.showa-u.ac.jp
}

Received 22 August 2020; Accepted 22 August 2020; Published 30 August 2020

Copyright ( $\odot 2020$ Kodai Tomioka et al. This is an open access article distributed under the Creative Commons Attribution License, which permits unrestricted use, distribution, and reproduction in any medium, provided the original work is properly cited.

In the article titled "Solid Pseudopapillary Neoplasm of the Pancreas with High-Grade Malignant Transformation Involving p16-RB Pathway Alterations" [1], author Yusuke Wada was affiliated to the Division of Gastroenterological and General Surgery, Department of Surgery, Showa University, Shinagawa, 1-5-8 Hatanodai, Shinagawa, 142-8666, Tokyo, Japan, which is incorrect. The corrected list of affiliations is shown above.

\section{References}

[1] K. Tomioka, N. Ohike, T. Aoki et al., "Solid pseudopapillary neoplasm of the pancreas with high-grade malignant transformation involving p16-RB pathway alterations," Case Reports in Surgery, vol. 2020, Article ID 5980382, 6 pages, 2020. 